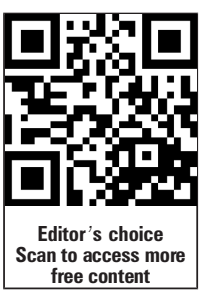

${ }^{1}$ San Diego Imaging, San Diego,

California, USA

2Department of Radiology, Winthrop-University Hospital Mineola, New York, USA ${ }^{3}$ Department of Radiology, Montefiore Medical Center, Bronx, New York, USA ${ }^{4}$ Department of Radiology, Medical College of Wisconsin, Milwaukee, Wisconsin, USA ${ }^{5}$ Insight Imaging, Roanoke, Virginia, USA

${ }^{6}$ North County Radiology, San Diego, California, USA

Correspondence to Dr Orlando Ortiz, Chairman, Department of Radiology, Winthrop-University Hospital, 259 First Street, Mineola, NY 11501, USA; oortiz@winthrop. org

Received 5 March 2012 Revised 15 May 2012 Accepted 20 May 2012

Published Online First 8 June 2012

\title{
Multicenter study to assess the efficacy and safety of sacroplasty in patients with osteoporotic sacral insufficiency fractures or pathologic sacral lesions
}

\author{
Keith Kortman, ${ }^{1}$ Orlando Ortiz, ${ }^{2}$ Todd Miller, ${ }^{3}$ Allan Brook, ${ }^{3}$ Sean Tutton, ${ }^{4}$ \\ John Mathis, ${ }^{5}$ Bassem Georgy ${ }^{6}$
}

\section{ABSTRACT}

Objective To assess the outcomes and safety after CTguided percutaneous sacroplasty in patients with painful sacral insufficiency fractures or pathologic sacral lesions. Methods A retrospective multicenter analysis of consecutive patients undergoing CT-guided sacroplasty for painful sacral insufficiency fractures or sacral lesions was undertaken. The inclusion criteria consisted of severe sacral pain not responding to conservative medical management with imaging evidence of unilateral or bilateral sacral insufficiency fractures or lesions. Outcome measures included hospitalization status (inpatient or outpatient), pre-treatment and posttreatment visual analog scale (VAS) scores, analgesic use and complications. Patients were followed at approximately 1 month and for at least 1 year after their sacroplasty procedure.

Results Two hundred and forty-three patients were included in the study, 204 with painful sacral insufficiency fractures and 39 with symptomatic sacral lesions. The average pre-treatment VAS score of $9.2 \pm 1.1$ was significantly improved after sacroplasty to $1.9 \pm 1.7$ in patients with sacral insufficiency fractures $(p<0.001)$. The average pre-treatment VAS score of $9.0 \pm 0.9$ in patients with sacral lesions was significantly improved after sacroplasty to $2.6 \pm 2.4(p<0.001)$. There were no major complications or procedure-related deaths. One patient who was treated for a sacral insufficiency fracture experienced radicular pain due to local extravasation of cement that subsequently required surgical decompression for symptomatic relief.

Conclusions CT-guided percutaneous sacroplasty is a safe and effective procedure in the treatment of painful sacral insufficiency fractures or lesions. It is associated with prompt and durable pain relief and should be considered as an effective treatment option in this patient population.

\section{INTRODUCTION}

The pain associated with an osteoporotic sacral insufficiency fracture or a pathologic sacral lesion can be severe and debilitating. Patients with sacral insufficiency fractures were historically treated with a combination of bed rest, analgesics and physical therapy. These injuries are termed insufficiency fractures, indicating that in these patients bone strength is not sufficient to withstand normal mechanical and kinetic forces. ${ }^{1}$ Sacral insufficiency fractures may be chronic at presentation due to a delay in diagnosis. Unlike osteoporotic vertebral compression fractures, sacral insufficiency fractures are not apparent on routine x-rays of the lumbosacral spine, yet sacral insufficiency fractures account for up to $4 \%$ of fractures of the spine and pelvis. ${ }^{2}$ As with osteoporotic vertebral compression fractures, these sacral insufficiency fractures predominantly occur in elderly women with osteoporosis. Advanced imaging techniques including CT, MRI and skeletal scintigraphy are often required to identify these sacral fractures. ${ }^{3}$ Patients with pathologic sacral lesions often undergo several diagnostic procedures including biopsy prior to being treated with surgery, aggressive surgical resection if they are a surgical candidate and/or radiation and chemotherapy and analgesic therapy. Despite these clinical interventions, there are patients who are either not surgical candidates or cannot undergo further chemotherapy or radiation therapy, but have disabling pain.

Patients with sacral low back pain and lesions that are refractory to conventional therapies are often subjected to prolonged hospitalization, extended care services and hospice care services. The impact of this sacral pathology on quality of life is devastating and the morbidity is significant. ${ }^{4} 5$

Sacroplasty is an augmentation procedure that involves the injection of acrylic bone cement into the fracture site of the sacrum in patients with osteoporotic sacral insufficiency fractures or focal lesions of the sacrum. The goal of sacroplasty is to obtain an analgesic effect by osseous stabilization in patients with pain related to lesions that weaken the sacrum. ${ }^{6}$ Sacroplasty is an extension of the vertebral augmentation procedure applied to the most caudal weight-bearing structure of the spinal axis. The first sacroplasty procedures reported in the literature were referred to as 'cementoplasty'. 78 The procedure subsequently has been called sacroplasty. ${ }^{9}$ Since those first publications, scattered reports in the literature have typically reported individual operator experiences with this procedure at one center. The purpose of this study is to accumulate and review the data from several centers where sacroplasty is performed in order to determine the current applications of this procedure, its efficacy and any complications that have been reported.

\section{METHODS}

This was a retrospective multicenter analysis of consecutive patients undergoing image-guided 
sacroplasty for painful sacral insufficiency fractures or painful sacral lesions.

The inclusion criteria consisted of severe sacral pain not responding to conservative medical management with imaging evidence (MRI, CT or skeletal scintigraphy) of unilateral or bilateral sacral insufficiency fractures, focal sacral mass or infiltrative sacral lesion. The presence of other pelvic or spinal fractures or lesions was also recorded. Furthermore, a patient's lower back pain symptoms had to be attributable to their sacral pathology as demonstrated on pain diagrams, interrogation and physical examination with or without fluoroscopic assistance. To be considered for sacroplasty a patient's sacral symptomatology had to be refractory to standard medical management. The latter consisted of any combination of bed rest, analgesics, partial weight bearing and use of orthosis. The procedure was not performed in those patients who were responding favorably to their conservative management or in those who had coexisting systemic or local infection. The latter, often consisting of sacral decubitus ulcers, was an absolute contraindication to performing sacroplasty. The procedure was also not performed in patients with uncorrected coagulopathy.

Operators who were trained and experienced in vertebral augmentation techniques performed the sacroplasty procedure. All procedures were performed using strict aseptic technique and pre-procedure intravenous antibiotic prophylaxis. Patients were placed prone and the procedures were performed using local lidocaine anesthesia along with intravenous sedation and analgesia. All patients were carefully monitored during and immediately after their procedures. All procedures were performed with CT, CT and fluoroscopy or CT fluoroscopy.

Short axis techniques with varying amounts of needle angulation were used depending on the size and extent of the lesion and the number of bone needles used (figure 1). With the short axis technique, a bone needle is advanced perpendicular to the dorsal surface of the sacral ala. The needle trajectory is also angled slightly medial to lateral in order to place the needle tip either within or adjacent to the fracture line. This maneuver also helps to avoid the sacral foramina. The short axis technique is ideally suited for CT guidance because the axial sectioning plane readily tracks the needle as it is advanced into the sacral ala (figure 2). The position of the needle relative to critical structures such as the sacral canal, sacral foramina, sacroiliac joint and the anterior sacral cortex is immediately demonstrated. The

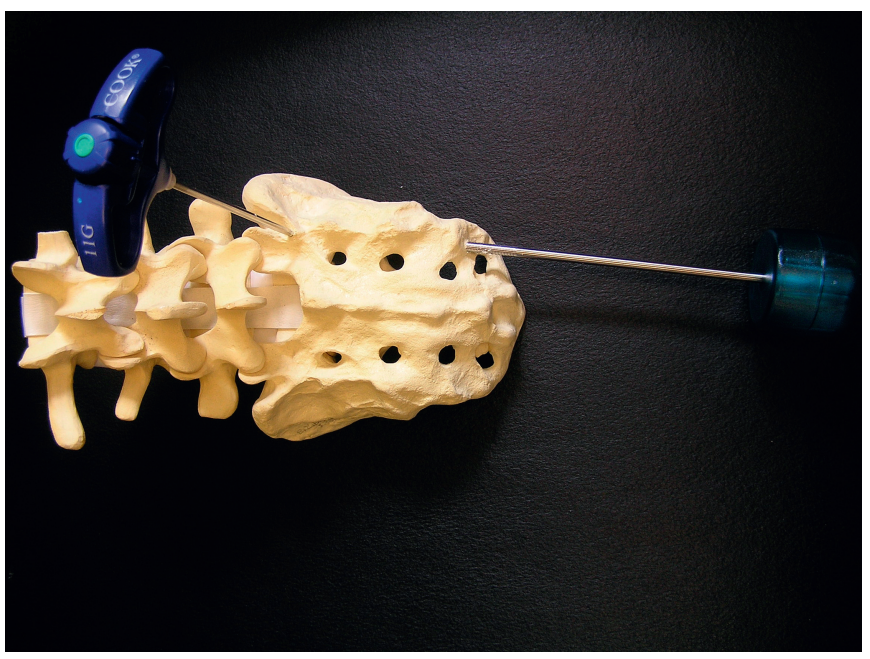

Figure 1 Photograph of sawbones model with needles in place for the short axis technique (flat-handle) and long axis technique. volume of injected cement, however, is sometimes limited by this approach. To compensate for this potential limitation, some operators will angle the needle slightly with an oblique orientation relative to the dorsal sacral surface. The placement of additional bone needles may also be necessary with this technique. The potential complications of the short axis technique include bleeding, infection, neural damage, dural tear and perforation of the anterior sacral cortex with hematoma formation or lumbosacral plexus injury, sacroiliac joint entry, cement extravasation into the sacral foramina or canal and cement embolus.

The other sacroplasty approach is the long axis technique (figure 1). With this technique the bone needle is advanced from the inferior and dorsal aspect of the sacral ala at S4 cephalad to the S1 level. The needle trajectory lies between the lateral margins of the sacral foramina and the sacroiliac joint. The long axis technique requires fluoroscopic guidance. The potential limitations of this technique are related to poor visualization of the sacral anatomic landmarks due to overlying bowel gas and osteoporosis. A larger injection of acrylic bone cement through a single bone needle is made possible with this technique. Nevertheless, the monitoring of cement injection with the long axis technique is challenging. The potential complications associated with bone needle placement and cement injection are similar to those that might occur with the short axis technique

One or more bone needles (11 or 13 gauge in size) were placed within the affected sacral ala or lesion using imaging guidance, avoiding the sacral canal and foramina as well as the anterior sacral cortex. The choice of entry sites was determined by the location of the fracture lines or the sacral lesion. Bone biopsy was performed prior to sacroplasty in those patients with a known or suspected neoplastic sacral lesion. Careful deposition of radiopaque polymethylmethacrylate or bioceramic cement was performed with imaging monitoring. The endpoints for termination of cement injection included satisfactory lesion filling or encroachment of cement upon the neural foramina or sacral canal (figure 2).

The number of bone needles (13 or 11 gauge), sides and sites of treatment and the volume of injected bone cement were documented. Outcome measures included hospitalization status (inpatient or outpatient), pre-treatment and posttreatment visual analog scale (VAS) pain scores, analgesic use including prescription narcotic or non-narcotic or over-thecounter pain medications. A change in pain score by $\leq 2$ points was deemed not significant in terms of pain profile improvement. The approximate average time between symptom onset and treatment evaluation was recorded. Patients were also monitored for major procedure-related adverse events including death, needle injury, cement extravasation, neurologic compromise, hemorrhage or infection. All patients were followed at approximately 1 month. Furthermore, all patients in the sacral insufficiency fracture group were followed up for at least 1 year after their sacroplasty procedure. The primary outcome was pain relief as reported at clinical follow-up by direct patient report and examination and documentation with a visual analog pain scale.

\section{RESULTS}

Of the 243 patients included in the study, 204 had osteoporotic sacral insufficiency fractures and 39 had focal mass or infiltrative lesions. One hundred and thirty-two patients were treated at the first institution, 21 patients were treated at the second institution, 38 patients were treated at the third institution, 21 
Figure 2 A man in his mid-60s with focal $7 / 10$ low back pain not responding to conservative management. MRI (not shown) showed extensive marrow edema within the left sacrum. (A) Axial CT image shows fracture line in left sacral ala. (B) Sacroplasty was performed using the short axis technique with an 11 gauge bone needle advanced obliquely through the fracture line within the left sacral ala. (C) Axial CT image obtained after the injection of $3 \mathrm{ml}$ acrylic bone cement shows the distribution of the cement within the fracture. The patient responded favorably to this procedure as evidenced by a follow-up pain score of $0 / 10$.
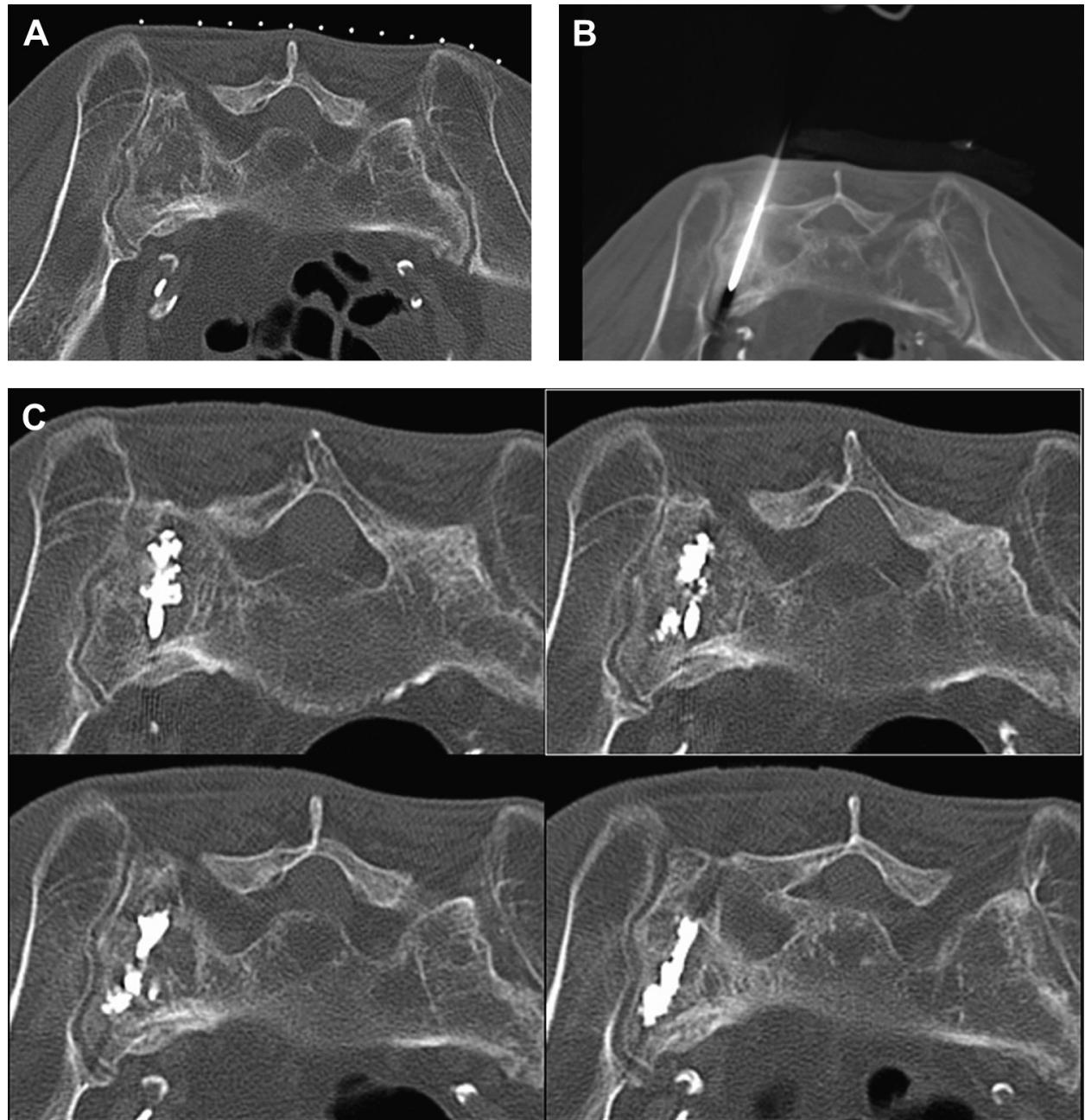

patients were treated at the fourth institution, four patients were treated at the fifth institution and 27 patients were treated at the sixth institution. Of those 204 patients with sacral insufficiency fractures, 176 patients were women and 28 were men with a mean age of 77.2 years. One hundred and sixty-nine patients had bilateral sacral insufficiency fractures and 35 had unilateral fractures. Sixty-five patients had additional fractures of the axial skeleton consisting of 39 pelvic fractures, 25 osteoporotic vertebral compression fractures of the thoracic or lumbar spine and one coccyx fracture. On average, patients were seen approximately 30.5 months after symptom onset. One hundred and eight patients were evaluated and treated as inpatients and 96 patients were managed as outpatients. An average of 2.5 bone needles was used for each procedure with a range of $1-5$. An average cement volume of $4.1 \mathrm{ml}$ was used for each procedure. Acrylic bone cement was used in 202 patients and bioceramic bone cement was used in two younger patients (ages 62 and 67). The average pre-treatment VAS score of $9.2 \pm 1.1$ was significantly improved after sacroplasty to $1.9 \pm 1.7$ in patients with sacral insufficiency fractures $(p<0.001$, two-tailed paired t test; table 1). The mean pain score before and after the procedure in patients with unilateral sacral insufficiency fractures was $8.7 \pm 1.5$ and $2.5 \pm 1.9$, respectively $(p<0.001)$. Furthermore, 64 of the 204 patients (31\%) reported complete pain relief. Marked reductions in analgesic use were observed, particularly in this latter group. Twenty biopsy procedures were performed in this group of patients, none of which demonstrated a neoplastic lesion. Seven of the 204 patients (3\%) did not experience major pain relief. Sacroplasty facilitated inpatient discharge in those patients who were already hospitalized. There were no procedure-related deaths, infections, pulmonary emboli or hemorrhages. One patient experienced radicular pain due to local extravasation of cement requiring surgical decompression for symptomatic relief. Another patient in this series developed progressive fracture dislocation 1 week after sacroplasty. Three patients with unilateral fractures were subsequently diagnosed with contralateral sacral insufficiency fractures; treatment of these fractures resulted in pain relief.

Of the 39 patients with focal mass or infiltrative sacral lesions, 25 were women and 14 were men with an average age of 68.9 years. Malignant lesions were diagnosed in 37 of the patients and confirmed by biopsy; 24 had metastatic disease (11

Table 1 Comparison of average pain scores before and after sacroplasty in patients with bilateral or unilateral sacral insufficiency fractures and in patients with underlying sacral lesions

\begin{tabular}{llll}
\hline Lesion type & $\begin{array}{l}\text { Pre-treatment } \\
\text { average VAS score }\end{array}$ & $\begin{array}{l}\text { Post-treatment } \\
\text { average VAS score }\end{array}$ & p Value \\
\hline $\begin{array}{l}\text { Bilateral sacral } \\
\text { insufficiency fracture }\end{array}$ & $9.2 \pm 1.1$ & $1.9 \pm 1.7$ & $<0.001$ \\
$\begin{array}{l}\text { Unilateral sacral } \\
\text { insufficiency fracture }\end{array}$ & $8.7 \pm 1.5$ & $2.5 \pm 1.9$ & $<0.001$ \\
$\begin{array}{l}\text { Sacral mass or } \\
\text { infiltrative lesion }\end{array}$ & $9.0 \pm 0.9$ & $2.6 \pm 2.4$ & $<0.001$ \\
\hline
\end{tabular}

The reduction in the average pain score after sacroplasty was statistically significant (two-tailed t test) in each patient group.

VAS, visual analog scale. 
with breast metastases), 11 patients had multiple myeloma and two had leukemia. Two patients had benign lesions, one with a hemangioma and the other with a cyst. Bilateral sacral lesions were identified in 15 patients; unilateral lesions were seen in 24 patients. Nineteen patients were evaluated and treated as inpatients and 20 patients were managed as outpatients. An average needle use of 1.7 per procedure was observed with a range of 1-4 needles per procedure. The average cement volume was $3.6 \mathrm{ml}$. The average pre-treatment VAS score of $9.0 \pm 0.9$ was significantly improved after sacroplasty to $2.6 \pm 2.4$ $(p<0.001$, two-tailed paired t test; table 1$)$. Seven of the 39 patients (18\%) reported complete pain relief. Overall, a marked reduction in use of analgesics was reported in this group of patients. Four of the 39 patients $(10 \%)$ did not experience significant pain relief. There were no procedure-related deaths, infections, pulmonary emboli, significant cement extravasations or hemorrhages in this patient group.

\section{DISCUSSION}

The sacrum is a weight-bearing structure that dissipates vertical axial forces that are transmitted along the spinal axis in a caudal direction. ${ }^{10}$ It functions as a keystone within the pelvic ring and is involved in stabilization of the pelvis as forces from the lower extremities are transferred cranially toward the sacrum via the pelvic girdle. Sacral lesions destabilize the pelvis resulting in pain that is aggravated by weight bearing including sitting, standing and walking. ${ }^{11}$ Pathologic processes that reduce bone strength in the sacrum include osteoporosis, mass lesions or infiltrative neoplasms within the sacrum. Osteoporotic sacral insufficiency fractures often extend vertically within the sacral ala and tend to parallel the sacroiliac joints along the lines of upward and downward force transmission through the sacrum. ${ }^{12-14}$ Load transfer related to weight bearing may play a significant role in their etiology. ${ }^{15}$ Benign or malignant neoplasms that occur within the sacrum similarly weaken or destroy the underlying supportive osseous matrix.

Sacral pathology due to a sacral insufficiency fracture or pathologic lesion can be a source of severe and debilitating low back pain. ${ }^{16}$ A delay in diagnosis is common in this patient population. ${ }^{17} 18$ The reasons for this diagnostic delay are multifactorial. First, the initial study that is often requested to evaluate patients with low back pain-a plain x-ray of the lumbar spine-is inherently insensitive to the presence of any sort of sacral pathology, especially in osteoporotic patients. ${ }^{3}$ MRI of the lumbar spine provides only limited views of the sacrum in the field of view. In other cases the imaging findings within the sacrum are so striking that the initial clinical concern is that of malignancy and not fracture. This occurred in several of our patients with sacral insufficiency fractures in whom a biopsy procedure was the initial requested medical test. Sacral insufficiency fractures often occur without any history of prior fall or trauma. These patients present with vague back pain symptoms and often have a prior history of cancer. ${ }^{3}$ Lastly, there is limited familiarity with the diagnosis of sacral insufficiency fracture among clinicians and radiologists.

Initial reports of the percutaneous injection of bone cement into the sacrum were for the treatment of painful sacral neoplasms. ${ }^{78}$ Once the feasibility of performing this procedure was proved, the technique was next applied as a treatment for a painful sacral insufficiency fracture that was unresponsive to conservative medical management. ${ }^{9}{ }^{19}$ All of these initial procedures were performed with fluoroscopic guidance, and all of these patients experienced pain relief through structural stabilization of the sacrum. ${ }^{20} 21$ Indeed, the use of computer models with finite element analysis shows that sacroplasty attenuates mechanical stress at the fracture site and decreases fracture gap micromotion. ${ }^{22-24}$

The reported clinical experience with sacroplasty has been somewhat limited. ${ }^{3} 4625$ This can be attributed to a relatively lower incidence of sacral fractures compared with osteoporotic vertebral compression fractures as well as the lack of clinician awareness of this relatively new procedure. ${ }^{21} \mathrm{~A}$ review of the medical literature revealed only anecdotal case reports and small retrospective case series. All of these early reports indicate that the procedure is feasible and offers the possibility of alleviating sacral pain in patients with osteoporotic or pathologic sacral lesions. ${ }^{19}{ }^{26-34}$ In one series of 13 patients with sacral insufficiency fractures treated under fluoroscopic guidance with prone oblique needle insertion, the authors reported that all 13 patients experienced subjective pain relief. There were no complications in this small study. ${ }^{30}$ Another small series of 13 patients treated with sacroplasty for painful sacral insufficiency fractures using a combination of CT and fluoroscopic guidance and prone oblique needle insertion showed moderate to complete pain relief in seven of the 11 patients who were available for shortterm follow-up. Five of the six patients who were available for long-term follow-up in this series were pain-free at the time of evaluation. These authors did not report any complications. ${ }^{29}$ Two other studies have reported pain scores before and after sacroplasty for painful sacral insufficiency fractures. In one study of 19 patients in which CT guidance and the long axis needle insertion technique were used, the initial mean VAS score of $8.0 \pm 1.9$ improved to $1.6 \pm 1.1$ at a 48 week follow-up interval. No complications were reported in this study. ${ }^{28} \mathrm{~A}$ recent prospective multicenter study addressed the relative efficacy of fluoroscopy-guided sacroplasty with posterior oblique needle insertion in the treatment of sacral insufficiency fractures in 52 patients. ${ }^{35}$ These authors reported significant improvements in mean VAS pain scores from 8.1 to 2.1 at 4 weeks and 0.8 at 52 weeks. Only one complication was reported in this group of patients, a case of transient $\mathrm{S} 1$ radiculitis that responded to a transforaminal epidural steroid injection.

Our study is a multicenter retrospective review of patients with both sacral insufficiency fractures and sacral lesions. Despite the retrospective nature, the common approach to performing the procedure with CT guidance and the similar approach to patient management and follow-up among the operators enabled us to combine the data for analysis. A greater number of patients with sacral pain had sacral insufficiency fractures $(n=204)$ compared with sacral lesions. ${ }^{36}$ A female predominance was observed in both patient groups, but especially in patients with sacral insufficiency fractures. Bilateral fractures were approximately four times more common than unilateral sacral insufficiency fractures. There was no significant difference in pain score improvement between treated bilateral and treated unilateral fractures. Many patients $(n=65)$ with sacral insufficiency fractures also had either pelvic or other osteoporotic vertebral compression fractures; the latter injuries undoubtedly contributed to the patient's back pain profile. The improvements in mean pain scores seen in the group with sacral insufficiency fractures (9.2 to 1.9) is similar to those reported in the previously mentioned studies. ${ }^{28} 35$ Complete pain relief was achieved in $31 \%$ of the patients. These effects were durable at the 1-year follow-up in the sacral insufficiency fracture group.

The average cement volume used to treat sacral insufficiency fractures was $4.1 \mathrm{ml}$. The two patients treated with bioceramic 
bone cement also experienced significant pain relief. The number of patients is not sufficient to make any conclusions about the potential advantages or disadvantages of bioceramic cement in this procedure. Certainly, the mix on demand feature of bioceramic cement is of theoretical benefit in a procedure where multiple needles are placed and careful slow cement injection is required. Bioceramic cement costs more than acrylic bone cement, but its use in middle-aged patients with sacral insufficiency fractures should be considered. Bioceramic cement does not appear to provoke an inflammatory response around its margins as has been reported in a few histologic bone specimens with polymethylmethacrylate. Bioceramic cement has biomechanical properties that are more similar to normal bone matrix, providing strength without dramatically increasing stiffness. The potential benefits of bioceramic cement will require further investigation.

A few complications occurred in the sacral insufficiency fracture group. One procedure-related complication of foraminal cement extravasation was observed $(0.05 \%)$. The procedure was unsuccessful in another patient with progressive fracture dislocation. Three patients $(1.5 \%)$ with treated unilateral sacral insufficiency fractures were diagnosed with subsequent fractures in the opposite ala within 3 months of their initial procedure. Patients with recurrent sacral pain after unilateral sacroplasty should therefore be evaluated for the possible occurrence of contralateral sacral fracture.

Results in patients with neoplastic or mass-like lesions in the sacrum were also favorable. The majority of these patients were diagnosed with either metastases or with multiple myeloma. The goal of sacroplasty in these patients was to provide palliative pain relief. ${ }^{31-34}$ The significant improvement in mean pain scores in our group of 39 patients from 8.9 to 2.6 is encouraging. Complete pain relief was possible in the two patients with benign but painful sacral lesions and in seven of the 39 patients $(18 \%)$ overall. In comparison, a greater percentage of patients in the neoplastic group (10\%) did not experience any major pain relief compared with patients in the sacral insufficiency fracture group $(3 \%)$. No major procedure-related complications were reported in any of the patients with sacral lesions. Long-term follow-up was not consistently available in this latter group of patients as a result of their primary neoplastic conditions.

The large number of patients included in this study enabled us to evaluate both the safety and the efficacy of CT-guided percutaneous sacroplasty. The initial reports that described this procedure indicated that imaging guidance with CT offers a safe approach to performing sacroplasty. ${ }^{26} 29 \quad 36-39$ Sacroplasty presents a unique set of challenges to the operator. ${ }^{6212540}$ It can be difficult to appreciate anatomic landmarks in osteoporotic bone with fluoroscopy. Moreover, the iliac bones of the pelvis prevent adequate visualization of the upper sacrum with lateral fluoroscopic evaluation. The pyramidal shape of the sacrum requires continuous multiple projections in order to localize instruments or implants within the sacrum. The oblique orientation of the neural foramina and convex course of the sacral canal complicates needle positioning and cement injection. Several potential complications may occur as a result of less than optimal anatomic visualization. ${ }^{4}$ These include local complications related to needle or cement injury such as radiculopathy due to compromise of the sacral canal, neural foramina or lumbosacral plexus or cement embolus from extravasation. Regardless of the modality used, it is essential that the cement is injected slowly and carefully in order to allow for visualization of opacified cement within the sacral ala. CT, whether used alone or in combination with fluoroscopy, dramatically improves visualization of the sacrum and the critical neural bearing structures within its substance. Our favorable results in this large multicenter study of sacroplasty patients are consistent with this observation.

\section{CONCLUSIONS}

Sacroplasty can be performed safely with precise delivery of acrylic bone cement into sacral insufficiency fractures and sacral masses using CT guidance. CT provides optimal visualization of fracture lines, precise percutaneous placement of bone needles and accurate monitoring and visualization of cement distribution. Complications of this procedure, in the literature and in our series, are rare. Sacroplasty can be an effective procedure in selected patients with debilitating pain due to either a sacral fracture or other sacral lesion; significant durable pain relief is seen in the majority of treated patients. Alleviation of sacral pain can facilitate the titration of analgesic therapy and early ambulation, impacting upon comorbid conditions such as decubitus ulcers and thromboembolic phenomena. CT-guided sacroplasty is associated with prompt and durable pain relief and should be considered a safe and effective treatment option in this patient population.

Contributors All authors have made substantial contributions to the conception and design of this project and manuscript and have provided the data stated in the Results section. All authors have participated in the drafting and redrafting of this manuscript and have approved the final version for submission.

\section{Competing interests None.}

Ethics approval Ethics approval was provided by the Institutional Review Boards at the respective institutions.

Provenance and peer review Not commissioned; externally peer reviewed.

Data sharing statement De-identified data sets, with full HIPAA compliance, were used to tabulate the overall data for the patients in this retrospective case series.

\section{REFERENCES}

1. Pentecost RL, Murray RA, Brindley HH. Fatigue, insufficiency, and pathologic fractures. JAMA 1964:187:1001-4.

2. Wat SY, Seshadri N, Markose G, et al. Clinical and scintigraphic evaluation of insufficiency fractures in the elderly. Nucl Med Commun 2007;28:179-85.

3. Lyders EM, Whitlow CT, Baker MD, et al. Imaging and treatment of sacral insufficiency fractures. AJNR Am J Neuroradiol 2010;31:201-10.

4. Gupta AC, Yoo AJ, Stone J, et al. Percutaneous sacroplasty. J Neurointervent Surg. Published Online First 15 Oct 2011. doi:10.1136/neurintsurg-2011-010136

5. Taillander J, Langue $\mathrm{F}$, Alemanni M, et al. Mortality and functional outcomes of pelvic insufficiency fracture in older patients. Joint Bone Spine 2003; 70:287-9.

6. Cho $\mathbf{C H}$, Mathis JM, Ortiz AO. Sacral fractures and sacroplasty. Neuroimaging Clin North Am 2010;20:179-86.

7. Marcy PY, Palussiere J, Descamps B, et al. Percutaneous cementoplasty for pelvic bone metastasis. Support Care Cancer 2000;8:500-3.

8. Dehdashti AR, Martin JB, Jean B, et al. PMMA cementoplasty in symptomatic lesions of the S1 vertebral body. Cardiovasc Intervent Radiol 2000;23:235-7.

9. Garrant M. Sacroplasty: a new treatment for sacral insufficiency fracture. J Vasc Interv Radiol 2002;13:1265-7.

10. Diel J, Ortiz 0, Katz D, et al. The sacrum: pathologic spectrum, multi-modality imaging and subspecialty approach. RadioGraphics 2001;21:83-104.

11. Finiels $\mathbf{H}$, Finiels PJ, Jacquot JM, et al. Fractures of the sacrum caused by bone insufficiency. Meta-analysis of 508 cases. Presse Med 1997;26:1568-73

12. Lin JT, Lane JM. Sacral stress fractures. J Womens Health (Larchmt) 2003; 12:879-88

13. Tsiridis E, Upadhyay N, Giannoudis PV. Sacral insufficiency fractures: current concepts of management. Osteoporos Int 2006;17:1716-25

14. Weber M, Hasler $\mathrm{P}$, Gerber $\mathrm{H}$. Insufficiency fractures of the sacrum. Twenty cases and review of the literature. Spine (Phila Pa 1976) 1993;16:2507-12.

15. Kayanja M, Tsai E, Yamashita T, et al. The biomechanics of insufficiency fractures and augmentation of the sacrum. Spine J 2006;6(Suppl):96.

16. Leroux JL, Denat B, Thomas $\mathrm{E}$, et al. Sacral insufficiency fractures presenting as acute low-back pain. Biomechanical aspects. Spine (Phila Pa 1976) 1993:18:2502-6.

17. Lourie H. Spontaneous osteoporotic fracture of the sacrum an unrecognized syndrome of the elderly. JAMA 1982;248:715-17. 
18. Grasland A, Pouchot J, Mathieu A, et al. Sacral insufficiency fractures: an easily overlooked cause of back pain in elderly women. Arch Intern Med 1996;156:668-74.

19. Pommersheim W, Huang-Hellinger F, Baker M, et al. Sacroplasty: a treatment for sacral insufficiency fractures. AJNR Am J Neuroradiol 2003;24:1003-7.

20. Belkoff SM, Mathis JM, Jasper LE, et al. The biomechanics of vertebroplasty: the effect of cement volume on mechanical behavior. Spine 2001;26:1537-41.

21. Kortman K, Mathis JM, Ortiz AO. Sacroplasty. In: Mathis JM, Deramond H, Belkoff SM, eds. Percutanoous Vertebroplasty and Kyphoplasty. 2nd edn. New York, NY: Springer, 2006:197-209.

22. Whitlow CT, Yazdani SK, Reedy ML, et al. Investigating sacroplasty: technical considerations and finite element analysis of polymethylmethacrylate infusion into cadaveric sacrum. AJNR Am J Neuroradiol 2007;28:1036-41.

23. Richards AM, Mears SC, Knight TA. Biomechanical analysis of sacroplasty: does volume or location of cement matter? AJNR Am J Neuroradiol 2009:30:315-17.

24. Anderson DE, Cotton JR. Mechanical analysis of percutaneous sacroplasty using C image based finite-element models. Med Eng Phys 2007;29:316-25.

25. Ortiz A0, Brook A. Sacroplasty. Tech Vasc Interventional Rad 2009;12:51-63.

26. Butler LC, Given CA, Michel SJ, et al. Percutaneous sacroplasty for the treatment of sacral insufficiency fractures. AJR Am J Roentgenol 2005:184:1956-9.

27. Smith DK, Dix JE. Percutaneous sacroplasty: long-axis injection technique. AJR Am J Roentgenol 2006;186:1252-5.

28. Kamel EM, Binaghi S, Guntern D, et al. Ouctome of long-axis percutaneous sacroplasty for the treatment of sacral insufficiency fractures. Eur Radiol 2009;19:3002-7.

29. Strub WM, Hoffmann M, Ernst RJ, et al. Sacroplasty by CT and fluoroscopic guidance guidance: is the procedure right for your patient? AJNR Am J Neuroradiol 2007;28:38-41
30. Jayaraman MV, Chang $\mathrm{H}$, Ahn SH. An easily identifiable anatomic landmark for fluoroscopically guided sacroplasty: anatomic description and validation with treatment in 13 patients. AJNR Am J Neuroradiol 2009;30:1070-3.

31. Uemura A, Matsusako M, Numaguchi $Y$, et al. Percutaneous sacroplasty for hemorrhagic metastases from hepatocellular carcinoma. AJNR Am J Neuroradiol 2005;26:493-5.

32. Atalay B, Caner H, Yilmaz C, et al. Sacral kyphoplasty for relieving pain caused by sacral hemangioma. Spinal Cord 2006;44:196-9.

33. Zhang J, Wu C, Gu Y, et al. Percutaneous sacroplasty for sacral metastatic tumors under fluoroscopic guidance only. Korean J Radiol 2008;9:572-6.

34. Wee B, Shimal A, Stirling AJ, et al. CT-guided sacroplasty in advanced sacral destruction secondary to tumour infiltration. Clin Radiol 2008;63:906-12.

35. Frey ME, DePalma JM, Cifu DX, et al. Percutaneous sacroplasty for osteoporotic sacral insufficiency fracutes: a prospective, multicenter, observational pilot study. Spine J 2008:8:367-73.

36. Heron J, Connell DA, James SL. CT-guided sacroplasty for the treatment of sacra insufficiency fractures. Clin Radiol 2007:62:1094-100.

37. Brook AL, Mirsky DM, Bello JA. Computerized tomography guided sacroplasty: a practical treatment for sacral insufficiency fracture. Spine (Phila Pa 1976) 2005:30:450-4.

38. Binaghi S, Guntern D, Schnyder P, et al. A new, easy, fast, and safe method for CTguided sacroplasty. Eur Radiol 2006;16:2875-8.

39. Layton KF, Thielen KR, Wald JT. Percutaneous sacroplasty using CT fluoroscopy. AJNR Am J Neuroradiol 2006;27:356-8.

40. Masala S, Konda D, Massari F, et al. Sacroplasty and iliac osteoplasty under combined CT and fluoroscopic guidance. Spine (Phila Pa 1976) 2006;31 E667-9. 